Meta

Journal des traducteurs

Translators' Journal

\title{
À propos d'une nouvelle traduction des Sonnets à Orphée
}

\section{Nestor Schumacher}

Volume 37, numéro 2, juin 1992

URI : https://id.erudit.org/iderudit/004056ar

DOI : https://doi.org/10.7202/004056ar

Aller au sommaire du numéro

Éditeur(s)

Les Presses de l'Université de Montréal

ISSN

0026-0452 (imprimé)

1492-1421 (numérique)

Découvrir la revue

Citer cet article

Schumacher, N. (1992). À propos d'une nouvelle traduction des Sonnets à

Orphée. Meta, 37(2), 232-242. https://doi.org/10.7202/004056ar d'utilisation que vous pouvez consulter en ligne.

https://apropos.erudit.org/fr/usagers/politique-dutilisation/ 


\section{À PROPOS D'UNE NOUVELLE TRADUCTION DES SONNETS À ORPHÉE}

NESTOR SCHUMACHER Waimes, Belgique

Les éditions Ulysse fin de siècle viennent de publier une nouvelle traduction des Sonnets à Orphée de Rainer Maria Rilke. Elle est due à Roger Lewinter ${ }^{1}$, qui avait déjà traduit les Élégies de Duino en 19842. C'est l'occasion pour nous de rapprocher cette traduction d'une cuvre célèbre et difficile de trois traductions antérieures: celle de Maurice Betz, publiée en $1938^{3}$, celle de J.-F. Angelloz, publiée en $1943^{4}$, et celle, plus récente, d'Armel Guerne'5.

Les Sonnets à Orphée sont datés du château de Muzot, où Rilke s'était retiré en 1921. C'est, au même titre que les Élégies de Duino, une ouvre de la maturité. Mais, alors que les Élégies avaient été commencées en 1912, c'est un même élan qui, au début de l'année 1922, inspira les Sonnets, écrits en quelques jours, et l'achèvement des Élégies. Le poète attribue ce renouveau à la découverte de Paul Valéry: «J'étais seul, j'attendais, toute mon œuvre attendait. Un jour, j'ai lu Valéry, j'ai su que mon attente était finie ${ }^{6} . »$

Mais l'impulsion immédiate des Sonnets vient d'un événement fortuit, la mort d'une très jeune danseuse, Véra Ouckama Knoop, que Rilke décrit dans une de ses lettres :

Cette belle enfant, qui commença par la danse et fit alors sensation auprès de tous ceux qui la virent, tant son corps et son âme exprimaient l'art inné du mouvement et de la transformation, déclara brusquement à sa mère qu'elle ne pouvait ni ne voulait plus danser... (c'était précisément au sortir de l'enfance) ${ }^{7}$...

La description par la mère de Véra de la maladie et de la mort de cette jeune artiste le trouble profondément, car il voit en elle l'exemple type de ces êtres jeunes qui se consument d'un seul coup et qui nous relient à la mort.

Son inachèvement et son innocence tiennent ouverte la porte de la tombe, si bien que, défunte, elle fait partie de ces puissances qui gardent la moitié de la vie fraîche et ouverte vers l'autre moitiế à la blessure béante ${ }^{8}$.

Le sous-titre des Sonnets à Orphée sera dès lors Geschrieben als Grab-mal für Wera Ouckama Knoop («Écrits comme monument funéraire pour Véra Ouckama Knoop»).

\section{ÉTUDE COMPARATIVE DES TRADUCTIONS DU SONNET 1.2}

Je me permets, dans cette première partie, de choisir ce sonnet, qui, s'il n'est pas le plus célèbre, a l'avantage de bien situer le thème initial de l'œuvre'?

Und fast ein Mädchen wars und ging hervor aus diesem einigen Glück von Sang und Leier und glänzte klar durch ibre Früblingsschleier und machte sich ein Bett in meinem Ohr. 
Und schlief in mir. Und alles war ihr Schlaf.

Die Bäume, die ich je bewundert, diese

fühlbare Ferne, die gefühlte Wiese

und jedes Staunen, das mich selbst betraf.

Sie schlief die Welt, Singender Gott, wie hast du sie vollendet, dass sie nicht begehrte, erst wach zu sein? Sieh, sie erstand und schlief.

Wo ist ihr Tod? O, wirst du dies Motiv erfinden noch, eh sich dein Lied verzehrte?Wo sinkt sie hin aus mir?... Ein Mädchen fast..

\section{PRESQUE UNE ENFANT...}

Presque une enfant, et qui sortait de ce bonheur uni du chant et de la lyre, et brillait, claire, dans ses voiles printaniers, et se faisait un lit dans mon oreille.

Elle dormait en moi. Tout était son sommeil. Les arbres jamais admirés, et ce sensible lointain, et le pré un jour senti, et tout étonnement qui me prenait moi-même.

Elle dormait le monde. Dieu poète, comment la parfis-tu pour qu'elle n'eût désir d'abord d'être éveillée ? Elle parut, dormit.

Où est sa mort? Ah! ce motif,

l'inventerai-je avant que mon chant se dévore?

Où sombre-t-elle, hors de moi ?.. Une enfant presque...

(Maurice Betz)

Et c'était presque une enfant, qui surgit de l'harmonie heureuse du chant et de la lyre et brilla, claire, à travers ses voiles printaniers et se fit un lit dans mon oreille.

Et qui dormait en moi. Et tout était son sommeil. Tous les arbres un jour admirés, ce lointain sensible, la prairie sentie et chaque étonnement qui m'atteignait moi-même.

Elle dormait le monde. Dieu chanteur, comment l'astu parfaite, pour qu'elle ne demandât pas à s'éveiller d'abord? Vois, elle naquit et dormit.

Où est sa mort? Ô, ce motif, pourras-tu

l'inventer encore, avant que ton chant ne se soit consumé ?... De moi elle s'échappe, où donc ?... Une enfant presque... 
Et presque elle était une enfant, qui jaillissait de l'unique bonheur du chant et de la lyre, claire et brillante sous ses voiles de printemps, et qui se prépara sa couche en mon oreille.

Et s'endormit en moi. Et tout fut son sommeil : les arbres dont jadis j'étais émerveillé, le sensible lointain, la prairie éprouvée, et chaque étonnement qui venait me frapper

Elle dormait le monde. Ô dieu-chanteur, comment l'as-tu parfaite afin qu'elle ignore le goût de s'éveiller d'abord? Vois : levée, elle dort.

Où est sa mort? Oh ! vas-tu, ce motif, le découvrir encore avant que ne soit consommé ton chant?

Où sombre-t-elle en me quittant ?... Une enfant presque...
\end{abstract}

(Armel Guerne)

Et presque une fille c'était et se dégageait de ce bonheur d'accord de lyre et de chant et transparaissait claire en ses voiles de printemps et se fit un lit dedans mon oreille.

Et dormit en moi. Et tout lui fut sommeil.

Les arbres que jamais j'admirai, ce lointain qui se sent, la prairie sentie, et chaque étonnement qui moi-même touchait.

Elle dormait le monde. Dieu qui chantes, que tu l'as accomplie, qu'elle ne convoitât pas être d'abord éveillée. Vois, suscitée, elle dormit.

Où est sa mort? Ô vas-tu, ce motif, encore l'inventer, avant que ta mélodie se dévore? Où s'exhale-t-elle de moi ?... Une fille presque...

(Roger Lewinter)

\title{
LA FORME DU SONNET
}

On sait que le sonnet a une forme fixe : il est composé de quatorze vers répartis en deux strophes de quatre vers sur deux rimes et une de six vers sur trois rimes. Le sizain est en pratique écrit en deux tercets, mais il s'agit bien, comme le montre l'agencement des rimes, d'une seule strophe.

Dans toute traduction de poème, il faut sacrifier quelque chose pour sauver ce que l'on considère comme l'essentiel. Ici, c'est la rime qui est sacrifiée malgré son 
importance dans la définition du sonnet et plus particulièrement dans le sonnet de Rilke, bâti sur des rimes embrassées.

Les traducteurs ont cependant compensé ce sacrifice par le recours à certains éléments phoniques :

- l'alternance, traditionnelle en poésie française, de finales masculines et féminines. Elle est presque systématique chez Betz et Angelloz, plus classiques, tandis que Guerne et Lewinter privilégient les finales masculines;

- 1'assonance: oreille/sommeil, sensible/sentie, désir/dormît (Betz) oreille/sommeil, l'as/pas (Angelloz) - oreille/sommeil, émerveillé /éprouvé, comment / chant (Guerne) - et surtout Lewinter (chant / printemps, oreille / sommeil, que / presque, encore / dévore);

- des rimes et des assonances intérieures comme : enfant/chant, mort/dévore (Betz) - sensible/sentie, naquit/dormit, mort/encore (Angelloz) - chant/brillante, ignore /d'abord, mort / encore (Guerne) - c'était / dégageait, jamais / sait, mort / encore (Lewinter).

Notons encore que les consonnes dominantes de l'original : $f / v$ se retrouvent, avec des modalités diverses, dans les quatre traductions.

Quant au rythme du sonnet, il repose chez Rilke sur une succession de quatre scansions avec des intervalles variables - du moins ici, car on a vanté à juste titre la multiplicité des mètres et des rythmes de ces 52 sonnets ${ }^{10}$.

Les traducteurs ont cherché à rendre ce rythme par un vers comprenant en général quatre mesures, mais avec des accommodements. Le plus régulier est A. Guerne, qui emploie presque uniquement le dodécasyllabe à quatre mesures. Chez Angelloz et Lewinter, le vers varie en nombre de syllabes et en mètres. Betz, grâce à une expression plus dense, obtient un rythme plus ramassé, de 8 à 12 syllabes, avec trois ou quatre mesures.

Le rapport entre le vers et le déroulement de la phrase est marqué dans la première strophe par la chaîne des und, qui la lie en un ensemble et y rattache le vers 5 . Betz a renoncé à cet effet, les autres traducteurs le maintiennent.

Par contre, ils ont traité différemment le problème des contre-rejets qui viennent couper ce rythme aux vers 6 et 9. diese / fühlbare Ferne et wie hast / du sie vollendet sont rendus tels quels par Angelloz, bien que le français ne permette pas l'accent tonique (ce / lointain sensible [...] comment l'as-/ tu parfaite). Betz cherche un compromis pour le premier (et ce sensible/lointain) et supprime le second. Gueme rend «ce» au premier et déplace la coupure pour le second (comment / l'as-tu parfaite). Lewinter fait de même et coupe sur un que exclamatif sur lequel nous reviendrons. Quant à l'enjambement du vers 12 - au sens spécifique que donne Jean Mazaleyrat à ce terme ${ }^{11}$ — Betz et Angelloz ne le soulignent pas, tandis que Guerne et Lewinter jouent sur la place de «encore» : (découvrir / encore - encore / l'inventer).

\section{LES CONVERGENCES}

Les convergences - parfois les concordances — s'expliquent d'abord par la volonté des traducteurs de rester aussi près que possible de l'original. Elles sont possibles lorsque la langue d'arrivée permet une traduction directe qui conserve la force expressive de la langue de départ. Ces passages sont ici des moments essentiels du poème.

Rappelons que le sonnet 1.1 est consacré au chant d'Orphée, que les animaux subjugués écoutent. Dans le Sonnet qui nous occupe, Véra, fille d'Orphée, s'identifie à son chat, qui s'installe dans l'oreille du poète. 
- und machte sich ein Bett in meinem Ohr (vers 4)

Si l'on met à part la traduction d'A. Guerne - qui s'explique par le choix d'un mètre régulier (dodécasyllabe), mais recourt à une image à mon sens trop concrète - la seule différence est le choix du temps : imparfait descriptif (Betz) ou passé simple pour la succession des impressions (Angelloz) ou encore passage de l'un à l'autre (Guerne et Lewinter).

\section{- und schlief in mir. Und alles war ihr Schlaf (vers 5)}

Ici, Angelloz passe à l'imparfait pour décrire l'état ressenti : tout, c'est-à-dire toute la beauté du monde, telle que le poète l'a éprouvée (les arbres... la prairie... les lointains...), est revécu à travers cette harmonie. Guerne et Lewinter soulignent l'aspect inchoatif.

- Sie schlief die Welt : Elle dormait le monde (vers 9)

Il y a concordance complète dans la traduction de cette proposition dont la force réside dans l'emploi transitif du verbe schlafen / dormir.

- Wo ist ihr Tod? : Où est sa mort? (vers 12)

Le texte par sa simplicité impose une traduction directe. Cette fois le terme de «mort» est prononcé, mais en même temps nié, car cette mort est présence dans le monde. On peut comparer au sonnet 1.26 à propos d'Orphée, déchiré par les Ménades: Dort singst du noch jetztz, Là, tu chantes maintenant encore.

\section{LES VARIANTES}

Les variantes sont naturellement nombreuses, conditionnées par le rythme choisi et les synonymes disponibles. Ne mentionnons que les écarts les plus significatifs dans un ensemble convergent, puisqu'il reflète une interprétation identique.

\section{— vers 1 et 14:Und fast ein Mädchen wars... Ein Mädchen fast}

Les trois premiers traduisent - sur un rythme chaque fois différent - par «presque une enfant»/ «une enfant presque», ce qui est suffisamment explicite. R. Lewinter, malgré les connotations possibles, préfère «fille», plus proche de Mädchen et rend ainsi à «presque» le sens de «presque... déjà» (et non «presque encore»).

- vers $1 / 2$ : hervorgehen a à la fois le sens d' «apparaître» (hervor) et de «sortir de» (gehen ...aus), d'où les variantes : sortir de (B), surgir de (A), jaillir de (G) et se dégager de (L), plus ambigu. Pour diesem einigen Glück (bonheur unique, dans le sens de «un»), Angelloz et Lewinter choisissent une transposition: «l'harmonie heureuse», «ce bonheur d'accord».

- vers 3: la difficulté réside dans glänzte durch avec l'arrêt rythmique sur klar. Angelloz donne une traduction littérale, Betz francise un peu (dans), Guerne transpose en adjectifs (claire et brillante), Lewinter a une solution originale (transparaissait dans).

- vers 6 à 8 : sans entrer dans tous les détails, on constate chez Lewinter le choix d'une construction qui s'écarte de la norme : le verbe à la fin de la proposition relative (que jamais j'admirai, qui moi-même touchait), là où les autres traducteurs obtiennent un effet analogue avec une relative à construction normale ou un groupe participial (les arbres jamais admirés, Betz). 
- vers $11:$ sie erstand und schlief

Erstehen est un verbe rare, auquel on peut donner le sens de aufstehen (se lever), auferstehen (ressusciter) et entstehen (naître, au sens figuré). De là des versions assez différentes par le sens et le rythme, la plus étonnante étant celle de Guerne (Vois, levée, elle dort). La plus fidèle au sens est celle d'Angelloz. Lewinter semble inspiré par l'étymologie (res / susciter), mais donne ainsi à l'expression un sens passif qui n'est pas dans erstand, mais s'harmonise avec le sens général.

\section{LES DIVERGENCES}

Elles sont légères, sauf peut-être la dernière.

- Et tout lui fut sommeil (Lewinter, vers 5): alors que les trois autres traducteurs parlent de «son sommeil», Lewinter interprète $i h r$ comme pronom personnel (lui, à elle), ce qui introduit une perspective différente; c'est pour le poète que tout est revécu dans le sommeil (la mort) de Véra, comme le montre le développement de la strophe 2;

- les vers 9 à 11 constituent une interrogation à nuance admirative. Les trois premiers traducteurs la rendent comme telle avec des différences dans le traitement du contrerejet et des nuances dans la traduction de nicht begehren (n'eût désir (B) - ne demandât pas (A) - ignore le goût (G)).

Mais $R$. Lewinter l'interprète comme une exclamation, supprime le point d'interrogation et introduit comme souvent par que. La succession d'un qui (relatif), d'un que (exclamatif) et d'un autre que (consécutif) est peu euphonique, mais semble destinée à freiner le lecteur dans la perception d'un texte très dense.

- vers 12 à 14 : le poète s'était adressé à Orphée dans le premier tercet. On s'attend donc à ce que la question du deuxième tercet s'adresse également à lui. En fait, cela provoque des difficultés d'interprétation déjà signalées par Angelloz dans ses commentaires. Par contre, si l'on suppose que le poète se parle à lui-même, on comprend sa crainte de voir le motif lui échapper avant que son œuvre soit réalisée. Ceci expliquerait également le passage difficile: wo sinkt sie hin aus mir? ainsi que le début du sonnet suivant (Ein Gott vermags. Wie aber, sag mir, soll / ein Mann ihm folgen durch die schmale Leier? Un dieu le peut. Mais comment, dis, l'homme le suivrait-il sur son étroite lyre?).

Seul Betz a levé cette ambiguïté en traduisant: Ah, ce motif,/l'inventerai-je avant que mon chant se dévore?

Wo sinkt sie hin aus mir? hinsinken aus marque à la fois l'éloignement, la séparation d'avec la jeune fille (hin, aus) et l'affaissement de celle-ci dans le sommeil de la mort. Le passage est rendu littéralement par Betz et paraphrasé par Guerne. La traduction d'Angelloz est ici moins imagée (s'échappe), celle de Lewinter suggère un mouvement ascendant, qui n'est pas dans sinken, mais conforme à l'esprit : identification du chant et du poème (s'exhale de moi).

Dans l'ensemble, Maurice Betz me semble avoir réussi une traduction assez heureuse, très proche de l'original, mais sur un rythme plus ramassé, avec une alternance régulière des finales, beaucoup d'assonances, quelques préciosités sans doute («la parfistu, qu'elle n'eût désir»), un traitement modéré des contre-rejets : une interprétation finale qui aide à la compréhension de l'ensemble.

A.-F. Angelloz est également fort proche du texte de Rilke, avec un rythme plus conforme à l'original, quelques transpositions («harmonie heureuse, tous les arbres, elle 
s'échappe»), mais sa grande fidélité à la forme extérieure, notamment quant aux contrerejets, donne une certaine impression d'étrangeté à la lecture.

A. Guerne adapte davantage le texte («claire et brillante, se prépara sa couche, s'endormit en moi, dont jadis j'étais émerveillé, ignore le goût, levée elle dort, en me quittant...»). Sa phrase est plus «normale», plus calquée sur le vers; il supprime ou atténue les contre-rejets. Le vers est régulier (en général dodécasyllabique).

Bien que les trois traducteurs précités restent dans l'ensemble assez proches du texte original, on retrouve ici les deux tendances fondamentales de la traduction littéraire: amener le lecteur vers l'œuvre étrangère ou adapter celle-ci au lecteur de la langue d'arrivée. Angelloz est le représentant du «dépaysement», Guerne de l'«adaptation», Betz me semble occuper une position intermédiaire, le conflit se jouant d'ailleurs aussi à l'intérieur de chaque traduction.

Quant à R.Lewinter, il donne une traduction également assez proche du texte bien qu'avec certaines libertés dans la transposition («ce bonheur d'accord, tout lui fut sommeil, suscitée, où s'exhale-t-elle»). La métrique est assez variable (9 à 14 syllabes), les assonances nombreuses, le traitement des contre-rejets équilibré. Mais on est frappé dans sa traduction par une syntaxe différente, qui calque ici la construction allemande ( $« E t$ presque une fille c'était, que jamais j'admirai, qui moi-même touchait»). Pour analyser cet aspect valablement, il me faut cependant élargir maintenant la perspective et prendre en compte l'ensemble des Sonnets.

À la lecture de la traduction de R. Lewinter, on est frappé par l'emploi d'une syntaxe particulière, qui s'écarte nettement de l'usage considéré comme «normal». Elle s'explique toutefois par les libertés toujours plus grandes prises par le langage de la poésie moderne, à la recherche d'un effet de surprise.

On peut relever les procédés suivants, illustrés par quelques exemples :

- la position finale du verbe conjugé, parfois en proposition principale, le plus souvent dans la proposition relative, et déjà notée dans l'analyse qui précède.

Sonnet I.7 Jamais la voix devant poussière ne lui défaut

(Nie versagt ihm die Stimme am Staube)

I.15 Elle s'est, délicieuse, à vous convertie.

(Sie hat sich köstlich zu euch bekehrt.)

avec le jus qui l'heureuse remplit! (mit dem Saft, der die glückliche füllt !)

II. 18 a preste sur les pans de la propre volte tracé?

(die... rasch an die Wandung der eigenen Wendung geschrieben?)

- l'inversion du sujet et du verbe :

I.18 Viennent annonciateurs qui l'exaltent

(Kommen Verkündiger die es erheben)

I.19 Ne sont point les souffrances reconnues

n'est point l'amour appris.

(Nicht sind die Leiden erkannt,

nicht ist die Liebe gelernt.)

- l'ellipse du verbe :

II.18 Cette innombrable chaleur, qui de toi ? (diese unzählige Wärme aus dir?) 
- l'ellipse de $s i$, avec un indicatif ou un subjonctif :

\author{
I.7 l'exemple divin l'étreint-il \\ (wenn ihn das göttliche Beispiel ergreift) \\ I.19 Le monde, fût-il preste mobile \\ (Wandelt sich rasch auch die Welt) \\ II.15 S'intercale une cruche \\ (Schiebt sich ein Krug ein)
}

- un emploi très personnel de $\grave{a}+$ infinitif tenant lieu de proposition relative :

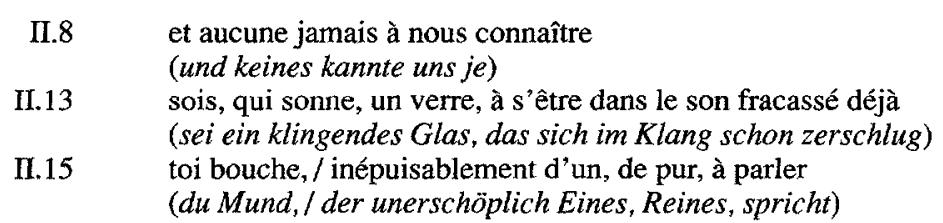

et, d'une manière générale, une rupture avec la construction habituelle. Citons un exemple typique et comparons-le à la traduction de J.-F. Angelloz :

II.16 Et l'agneau son grelot réclame de par, qui plus impassible, l'instinct.

Angelloz: Alors que l'agneau demande sa clochette, poussé par un instinct plus paisible. (Und das Lamm erbittet seine Schelle aus dem stilleren Instinkt.)

La plupart des procédés relevés ci-dessus se recoupent de façon frappante avec la description que fait Hugo Friedrich de la «nouvelle langue» de la poésie moderne dans «Die Struktur der modernen Lyrik» - spécialement dans le chapitre «Weiteres zur neuen Sprache», en se fondant surtout sur une analyse approfondie de l'œuvre de Rimbaud et de Mallarmé12.

D'autre part, il saute aux yeux que certains de ces procédés ont une parenté directe avec la construction allemande. Ils permettent donc, en principe, une traduction plus proche de l'original. Mais une rapide comparaison avec le texte de Rilke, cité entre parenthèses, montre que c'est loin d'être toujours le cas. Ils sont donc employés pour d'autres motifs : recherche d'originalité ? volonté d'échapper à une traduction trop fluide, pour arrêter le lecteur sur un texte très dense? Toujours est-il que ces ruptures provoquent chez le lecteur l'effet de surprise recherché par la poésie moderne. Mais, du point de vue fidélité, on peut se demander si ce bouleversement de la syntaxe traditionnelle est justifié ici, car Rilke, s'il a «travaillé contre le courant de la langue», respecte en général le cadre de la phrase littéraire allemande, comme on peut d'ailleurs le constater par les textes cités.

Il est cependant un autre aspect essentiel où l'effort de renouveau me paraît plus conforme au langage rilkéen, c'est le travail sur le vocabulaire. Celui-ci se trouve renouvelé par les procédés suivants :

- l'emploi fréquent de l'adjectif substantivé au sens neutre, comme en allemand:

I.13 Pour un dieu un aisé (für den Gott ein Leichtes)

I.14 D'obscur monte... (Aus Dunkel steigt...)

I.18 Entends-tu le nouveau? (Hörst du das Neue?)

I.19 accomplie, toute chose/à l'archaïque fait retour (alles Vollendete fällt/ heim zum Uralten) 
- le passage d'une catégorie à l'autre, notamment l'emploi d'infinitifs substantivés, qui forment éventuellement des groupes nominaux:

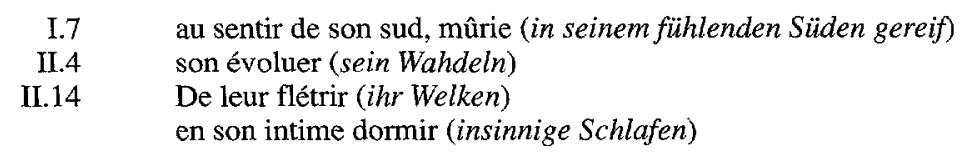

- l'emploi de mots rares ou de termes désuets :

I.17 dire de chenus (Spruch von Ergrauten)

II.2 des lumières serves (der dienenden Lichter)

II.15 fluent (fließend)

II.18 la propre volte (der eigenen Wendung)

I.17 de tout issant / racine (all der Erbauten/Wurzel)

II.23 et trop vieux pour ce qui onc ne fut (und zu alt für das, was niemals war)

II.26 Dans les entre-deux d'espaces / d'icelui, de l'espace du ciel

(in Zwischenräume / dieses des Weltraums)

- la formation de dérivés originaux:

II.19 dicible (säglich)

II.27 brisables (zerbrechlich)

I.1 Ô pur surmontement ! (O reine Uebersteigung!)

- la formation de nombreux composés sur le modèle rilkéen :

I.15 son être-suave (ihr Süßsein)

II.5 étoile-fleur (Blütenstern)

II.10 l'être-là (das Dasein)

II.11 de notre être-endeuillé cheminant... (unseres wandernden Trauerns), etc.

Il y a, selon $\mathrm{H}$. Friedrich, une forte tendance nominale dans la poésie moderne. Le substantif est mis en valeur au détriment du verbe. Contribuent à cette mise en évidence du nom:

- la suppression de l'article défini, que l'on peut observer dans plusieurs exemples cités, également dans

I.14 Nous en usons avec fleur, vigne, fruit.

(Wir gehen um mit Blume, Weinblatt, Frucht.)

II.12 De séparation est enfant ou neveu tout espace heureux

(Jeder glückliche Raum ist Kind oder Enkelvon Trennung)

- et les nombreuses antépositions du complément du nom, comme :

II.15 der Aquädukte Herkunft (des aqueducs la provenance)

Deux des traducteurs de Rilke avaient insisté dans leur introduction sur cette lutte avec la langue. A.-F. Angelloz écrivait que les Élégies et les Sonnets «révèlent, en particulier, plus qu'aucun autre poème allemand, un combat avec la langue, dont Rilke ne sortit vainqueur qu'en reculant à l'extrême les possibilités qu'elle offre ${ }^{13}$ ». De son côté, Maurice Betz, le traducteur des Cahiers de Malte Laurids Brigge, qui a encore connu le poète - insiste dans un «Avertissement du traducteur» :

Cependant, une grande prudence s'imposait dans une telle tentative de traduction rythmée. Ainsi que Rilke l'a lui-même justement observé, la langue française «académise» parfois dangereusement les poèmes étrangers soumis à son empreinte, et si l'on veut échapper à ce 
risque, il faut «travailler contre le courant de la langue», voire même «entrer en lutte avec elle $\gg 14$.

Roger Lewinter semble avoir suivi ces conseils. Il s'est inspiré, pour ce faire, des acquis de la poésie moderne - surtout depuis Mallarmé —, mais aussi des structures de la langue allemande et des recherches de Rilke. Il contribue ainsi comme traducteur à l'enrichissement et à l'assouplissement du langage poétique et donne des Sonnets une traduction qui rend un son neuf.

Pour sortir du fragmentarisme auquel nous a obligé l'analyse, nous voudrions, pour terminer, livrer au lecteur, et ce sans commentaires, la traduction par R. Lewinter d'un des plus beaux poèmes de ce recueil, le célèbre sonnet I.15, intitulé parfois Dansez l'orange ${ }^{15}$.

Wartet..., das schmeckt... Schon ist auf der Flucht.

... Wenig Musik nur, ein Stampfen, ein Summen-.

Mädchen, ihr warmen, Mädchen, ihr stummen,

tanzt den Geschmack der erfahrenen Frucht!

Tanzt die Orange. Wer kann sie vergessen, wie sie, ertrinkend in sich, sich wehrt wider ihr Süssein. Ihr habt sie besessen. Sie hat sich köstlich zu euch bekehrt.

Tanzt die Orange. Die wärmere Landschaft, werft sie aus euch, dass die reife erstrahle in Lüften der Heimat! Erglühte, enthüllt

Düfte um Düfte. Schafft die Verwandtschaft mit der reinen, sich weigernden Schale, mit dem Saft, der die glückliche füllt!

Attendez... Cela a le goût... Déjà il est en fuite.

... Fort peu de musique, un piétinement, un bourdonnement - :

$\dddot{\hat{O}}$ vous, jeunes filles ardentes, $\hat{o}$ vous, jeunes filles muettes, dansez le goût du fruit éprouvé !

Dansez l'orange. Qui peut oublier comment, se fondant en elle-même, elle se défend contre sa propre douceur. Vous l'avez possédée. À vous, ô délices! elle s'est convertie.

Dansez 1'orange. Le paysage plus chaud, projetez-le hors de vous, qu'elle rayonne de maturité dans les airs de son pays! Embrasées, dévoilez

un à un ses parfums ! Créez la parenté avec l'écorce pure qui se refuse, avec le suc qui emplit l'heureuse! 
Attendez..., c'est saveur... c'est fuyant déjà.

... Que peu de musique, un trépignement, un bourdonnement -

Filles, chaudes vous, filles, muettes vous,

dansez la saveur du fruit en son expérience !

Dansez l'orange. Qui peut l'oublier

comme elle, qui se noie en elle-même, se défend

contre son être-suave. Vous l'avez possédée.

Elle s'est, délicieuse, à vous convertie.

Dansez l'orange. Le paysage plus chaud, de vous lancez-le, que, mûre, elle irradie dans des brises natales. Embrasées, dévoilez

parfums sur parfums. Créez la parenté avec l'écorce pure qui se refuse,

avec le jus qui l'heureuse remplit!

Notes

1. Rainer Maria Rilke: Les Sonnets à Orphée, écrits comme un tombeau pour Veira Ouckama Knoop, traduction française de Roger Lewinter, Dijon, 1987.

2. Rainer Maria Rilke: Les Elégies de Duino, traduction de Roger Lewinter, éd. G. Lebovici, 1984.

3. Rainer Maria Rilke: Poésie, traduction de Maurice Betz, Paris, 1938.

4. Rainer Maria Rilke: Duineser Elegien. Die Sonette an Orpheus. Les Élégies de Duino. Les Sonnets à Orphée, traduits et préfacés par J.-F. Angelloz, Paris, 1943.

5. Rainer Maria Rilke: Les Elégies de Duino. Les Sonnets à Orphée; traduits de l'allemand par Armel Guerne, édition bilingue, Paris, 1972.

6. Monique Saint-Hélier: Ȧ Rilke pour Nö̈l, p. 21, cité par J.-F. Angelloz, op. cit., p. 11.

7. Lettre à la comtesse Sizzo-Noris Crouy, cité par J.-F. Angelloz, p. 12.

8. Rainer Maria Rilke: Lettres de Muzot (1921-1926), p. 338, cité par J.-F. Angelloz, p. 13

9. Traduction de M. Betz, op. cit., p. 223; J.-F. Angelloz, p. 15; A. Gueme, p. 107; R. Lewinter, p. 12. Texte de Rilke, in J.-F. Angelloz, p. 144.

10. Wolfgang Leppmann: Rilke, sa vie, son curre, Paris, 1984, p. 333.

11. Jean Mazaleyrat: Éléments de métrique française, Paris, Armand Collin, Collection «U2», 1974, p. 127.

12. Hugo Friedrich: Die Struktur der modernen Lyrik, Erweiterte Neuausgabe, Hambourg, 1974-1976.

13. J.-F. Angelloz: op. cit., p. 33.

14. M. Betz: op. cit., pp. $41-42$

15. Texte de Rilke, in J.-F. Angelloz, op. cit., p. 170, traduction de R. Lewinter, op. cit., p. 25. 